\title{
石墨烯的光化学修饰方法

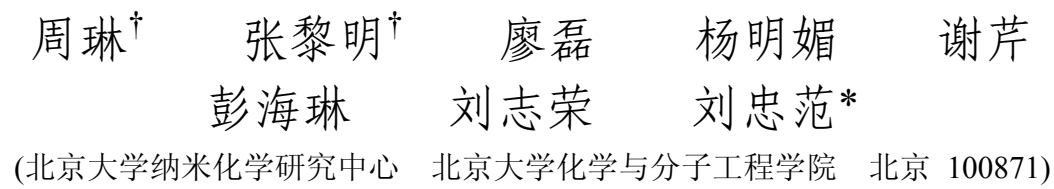

\begin{abstract}
摘要 石墨烯是由 $\mathrm{sp}^{2}$ 杂化碳原子组成的具有蜂窝状结构的二维原子晶体. 石墨烯的共价化学修饰是石墨烯研究领域 的一个新的热点, 也是石墨烯材料的表面改性和能带调控、以及合成新型二维石墨烯衍生物的重要途径. 完整的二维 蜂窝结构和离域大 $\pi$ 键使得石墨烯的化学性质非常稳定, 难以通过常规的化学反应获得高效的表面修饰, 这是石墨烯 共价化学的主要挑战. 近年来, 我们发展了一系列基于光化学原理的石墨烯共价修饰方法, 利用光化学过程产生的活 性自由基实现了石墨烯的高效共价加成和氧化反应, 为石墨烯的光化学能带工程奠定了理论和实验基础. 本文将以这 些研究成果为主线, 系统地阐述石墨烯的光化学修饰方法及其二维反应特性, 并对该领域的未来发展趋势和所面临的 挑战进行简要的展望.
\end{abstract}

关键词 石墨烯; 光化学; 自由基; 光氯化; 光催化; 非对称修饰

\section{Photochemical Modification of Graphene}

\author{
Zhou, $\operatorname{Lin}^{\dagger}$ \\ Zhang, Liming ${ }^{\dagger}$ \\ Liao, Lei \\ Yang, Mingmei \\ Peng, Hailin \\ Liu, Zhirong \\ Liu, Zhongfan* \\ Xie, Qin \\ (Center for Nanochemistry, College of Chemistry and Molecular Engineering, Peking University, Beijing 100871)
}

\begin{abstract}
Graphene, a two-dimensional (2D) atomic crystal composed of single-layer hexagonal mesh of carbon atoms, is one of the most exciting materials being investigated today. Graphene chemistry, the covalent functionalization of graphene as a giant molecule, provides a promising approach to controllably engineer graphene's band structure, create novel graphene derivatives and tailor the interfacial characteristics. One of the great challenges for graphene functionalization originates from its strong chemical stability, thus highly reactive chemical species are needed as the reactants. In recent years, we have been working on the photo-induced free radicals-based photochemistry of graphene, targeting the efficient graphene functionalization for its band structure engineering. Various photochemical modification methods have been developed, such as photochemical chlorination, photochemical methylation, photocatalytic oxidation and bifacially asymmetric functionalization of graphene. The homogeneous and nondestructive photochlorination of graphene could remove the conducting $\pi$-bands and open up a band gap in graphene. $\mathrm{TiO}_{2}$-based photocatalytic oxidation of graphene could realize photochemical tailoring of graphene, including ribbon cutting, arbitrary patterning on any substrate, layer-by-layer thinning, and localized graphene to graphene oxidation conversion. Using photochemical reaction of graphene as a probe, we have investigated the dimension effects on graphene chemistry, including the thickness, stacking order, single- and double-side, and edge dependent reactivity in graphene. After two-step functionalization of graphene, we have fabricated the thinnest Janus disc named Janus graphene, which comprises two kinds of decorations separated by the one-atom-thick carbon layer. It is found that chemical decorations on one side are capable of affecting both chemical reactivity and wettability of the opposite side, indicative of communication between the two grafted decorations separated by a single-layer graphene. In this review, we select several typical examples to demonstrate such kinds of photochemical graphene engineering and its intrinsic $2 \mathrm{D}$ reaction characteristics, together with a brief discussion on the future directions, challenges and opportunities in this research area.
\end{abstract}

Keywords graphene; photochemistry; free radical; photochlorination; photocatalysis; asymmetric functionalization

\section{1 引言}

石墨烯, 由碳原子以 $\mathrm{sp}^{2}$ 杂化方式构成的六方密堆 蜂巢结构的单原子层, 是人类最早发现的二维原子晶 体. 2004 年, 英国物理学家 Geim 和 Novoselov 借助普通
胶带，通过机械剥离方法首次获得了独立存在的石墨 烯 ${ }^{[1]}$. 石墨烯的发现推翻了 20 世纪 30 年代朗道和皮尔 斯提出的二维晶体由于热力学不稳定性而不存在的论 断 ${ }^{[2,3]}$, 开启了二维原子晶体的新时代. 石墨烯研究领域 的迅猛发展归因于石墨烯超出其他材料的神奇性质，有

* E-mail: zfliu@pku.edu.cn ${ }^{\dagger}$ These authors contributed equally to this work.

Received August 29, 2013; published October 25, 2013.

Project supported by the National Basic Research Program of China (Nos. 2013CB932606, 2012CB933404, 2011CB933003) and the National Natural Science Foundation of China (Nos. 51121091, 51290272).

项目受科技部国家重点基础研究发展规划(Nos. 2013CB932606, 2012CB933404, 2011CB933003)和国家自然科学基金(Nos. 51121091, 51290272)资助. 
些甚至达到理论预测的极限, 例如, 室温下的电子迁移 率为 $2.5 \times 10^{5} \mathrm{~cm}^{2} \cdot \mathrm{V}^{-1} \cdot \mathrm{s}^{-1}$ (理论极限 $2 \times 10^{5} \mathrm{~cm}^{2} \cdot \mathrm{V}^{-1}$ ・ $\left.\mathrm{s}^{-1}\right)^{[4,5]}$; 能承载超高的电流密度 (超出铜 100 万倍 $)^{[6,7]}$; 高热导率(超过 $\left.3000 \mathrm{~W} \cdot \mathrm{mK}^{-1}\right)^{[8]} ; 1 \mathrm{TPa}$ 的杨氏模量, 本 征强度达 $130 \mathrm{GPa}^{[9]}$, 接近理论预测极限 ${ }^{[10]}$; 光吸收率 极低 $(\sim 2.3 \%)^{[11]}$; 对所有气体均不通透 ${ }^{[12]}$. 这一单原子 层厚度的碳晶体独特地结合了超高的导电性、导热性及 优异的机械性能、不通透性和其他不同于常规材料的特 殊性质, 是诸多应用领域如高频电子器件 [13]、光电器 件 ${ }^{[14]}$ 、生物和气体传感 ${ }^{[15]}$ 、透明导电薄膜 ${ }^{[16]}$ 等的下一代 首选材料.

迄今为止, 人们已经发展出多种石墨烯的制备方 法, 如机械剥离法 ${ }^{[1,17]}$ 、化学剥离法 ${ }^{[18,19]}$ 、石墨氧化物还

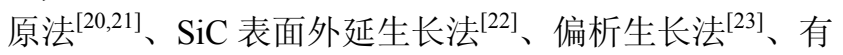
机合成法 ${ }^{[24]}$ 和化学气相沉积法 ${ }^{[25 ~ 28]}$. 其中化学气相沉 积法被认为是获得大面积高质量石墨烯的最有前景的 方法，已实现了在多种金属及合金基底 $(\mathrm{Cu}, \mathrm{Ni}, \mathrm{Co}$, $\mathrm{Mo}-\mathrm{Ni}$ 等)上生长高质量 (载流子迁移率达 45000 $\mathrm{cm}^{2} \cdot \mathrm{V}^{-1} \cdot \mathrm{s}^{-1}$ )、大面积(可达 30 英寸)、层数可控的石墨 烯 ${ }^{[16,25,29 ~ 33]}$. 石墨烯的共价化学, 即通过共价反应在石 墨烯骨架上引入各种化学官能团, 在制备石墨烯衍生 物、石墨烯的表界面改性、化学掺杂和能带调控以及制 备石墨烯复合材料等诸多方面具有重要意义. 石墨烯是 无能隙的半金属材料 ${ }^{[34]}$, 不能满足逻辑器件的电流关 断要求, 因而打开带隙是石墨烯电子学的主要挑战之 一. 已报道的调控石墨烯带隙的主要方法包括: 基于量 子限域效应的石墨烯纳米带 ${ }^{[35 ~ 38]}$ 和纳米网结构 ${ }^{[39,40]}$; 对 $\mathrm{AB}$ 堆垛的双层石墨烯施加垂直不对称场 ${ }^{[41 ~ 45]}$; 以及 通过共价化学修饰打开带隙 ${ }^{[46,47]}$ 等. 其中共价化学修饰 会导致石墨烯中的碳原子由 $\mathrm{sp}^{2}$ 杂化转变为 $\mathrm{sp}^{3}$ 杂化, $\pi$ 键对应的导带消失从而打开带隙 ${ }^{[48]}$, 被认为是一种成 本低廉、操作便利和可大规模应用的调控石墨烯能带的 方法. 石墨烯也是发展新型二维材料的平台, 在碳骨架 结构上接入其它官能团可以制备新的石墨烯衍生物. Geim 小组 ${ }^{[49]}$ 通过氢化和氟化分别制得二维石墨烯衍生 物晶体石墨烷和氟化石墨烯 ${ }^{[50,51]}$, 此类二维新材料属于 宽带隙半导体 $(>3 \mathrm{eV})$, 在室温下保持稳定. 通过周期 性的氢化和重氮盐反应, Tour 小组 ${ }^{[52]}$ 制备了石墨烯超晶 格材料. 石墨烯的表界面性质会因为引入的修饰基团而 显著改变. 通过在石墨烯表面引入不同性质的官能团, 可以得到均匀分散的石墨烯水相 ${ }^{[53]}$ 和油相 ${ }^{[54]}$ 溶液, 推 动了石墨烯的溶液化学和实际应用 ${ }^{[53]}$. 化学修饰还可

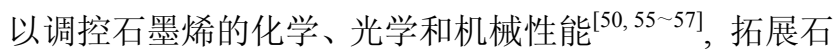

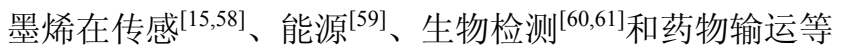
诸多领域的应用 ${ }^{[62 ~ 64]}$. 此外, 石墨烯本质上是二维的芳 香性稠环大分子, 它将化学反应限域在二维平面上, 使 得化学反应与维度产生关联, 是研究二维化学反应, 探 讨维度对化学反应行为和反应机理的影响的理想模型.
利用石墨烯的共价化学反应作为探针, 可以研究二维石 墨烯分子的独特化学性质. 总之, 共价化学修饰可以极 大地丰富和拓展石墨烯的研究内涵，因此，发展高效的 共价化学修饰方法至关重要.

由于石墨烯的化学惰性和热力学稳定性, 对其高效 的共价修饰依然困难重重. 石墨烯的化学惰性源于其均 匀且高度离域的电子分布. 一方面, 石墨烯表面不存在 弱键或者不稳定的键、悬挂键、高度局域的轨道或电荷 等常发生化学反应的高活性位点. 本征石墨烯没有曲 率，且表面无官能团的特点也导致其缺乏反应活性位 点. 另一方面, 石墨烯中每个碳原子是三重对称的 $\mathrm{sp}^{2}$ 杂化, $\pi$ 轨道延伸出碳原子平面, 形成自钝化的、稳定的 大共轭体系. 共价反应需打破该稳定的大 $\pi$ 共轭体系, 不仅在热力学上不利, 并且反应中涉及到的在成键碳原 子附近形成高能自由基过程也是能量不利的. 此外, 石 墨烯经共价修饰后, 碳原子由 $\mathrm{sp}^{2}$ 杂化转变为 $\mathrm{sp}^{3}$ 杂化会 导致石墨烯碳骨架形变, 由此带来的高反应能垒也导致 石墨烯共价修饰困难. 因此, 石墨烯表面有效的共价反

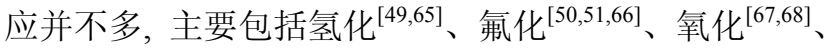
重氮盐加成 ${ }^{[69]}$ 及环加成反应 ${ }^{[70]}$. 亟需发展新的、高效的 石墨烯共价修饰方法, 引入不同功能和种类的官能团, 实现石墨烯的电学、光学、化学等诸项性质的可控调节.

光化学反应是物质在光照射下而发生的化学反应. 基态分子吸收光子后，从基态向激发态跃迁. 激发态通 常有三种, 分别为解离激发态、高能激发态和较稳定激 发态. 当吸收的光子能量大于或等于分子内某化学键的 解离能时, 分子会直接解离. 波长为 $250 \mathrm{~nm}$ 附近的紫外 光提供的能量足以使有机化合物中的几乎所有类型的 单键解离 ${ }^{[71]}$. 光解离是最基本的光化学过程, 它可以导 致处于电子激发态的分子发生光化学反应. 依据光化学 第一定律，当入射光波长范围与分子吸收光波长范围有 重叠且能量高于键能时, 则可使吸光分子断键产生自由 基. 因此，选择合适的光源对于光化学反应尤为重要. 自由基是带有不成对电子的原子或基团，具有高反应活 性，能够克服石墨烯的惰性及高反应能垒，是与石墨烯 发生共价反应的理想物种. 光引发是产生自由基的常用 方法, 选择高活性的光生自由基与石墨烯发生共价反 应，可望实现石墨烯高效的共价化学修饰，同时对其结 构及性质进行调控. 这就是我们提出的石墨烯的光化学 修饰和光化学能带工程的理论基础. 如图 1 所示, 中性 分子在光照下产生自由基 $\mathrm{X} \cdot \mathrm{X} \cdot \mathrm{X}$ 与暴露的石墨烯表面反 应，实现光诱导的石墨烯共价修饰. 基于自由基的石墨 烯光化学反应有利于实现高效可控的化学修饰, 并有着 诸多的优点: (1)光激发分子断键产生的自由基往往带有 剩余能量，活性很高，容易与惰性石墨烯反应. 且利用 光引发自由基不依赖于高温环境，甚至可以在低温下进 行，对石墨烯表面没有破坏作用; (2)在复杂体系中，依 据不同分子的吸收光谱, 可以选择不同波长的光源选择 
性引发反应，控制光化学反应的进程; (3)控制光功率密 度，可以调控自由基的浓度，从而控制化学反应的速率; (4)利用光易于图形化的特点, 通过光掩模技术, 易于实 现石墨烯表面定域的图案化共价修饰; (5)石墨烯的光化 学自由基反应很多都是气相反应, 不涉及溶液处理过 程，与已有石墨烯的半导体加工工艺兼容; (6)光引发的 高活性自由基种类繁多, 具有不同的化学活性和功能, 通过光化学反应可以实现石墨烯的各种功能化修饰.

在过去几年里, 我们发展了多种石墨烯的光化学修 饰方法，包括光氯化加成反应、光甲基化加成反应、光 催化氧化反应、以及双面非对称加成反应等, 系统地考 察了层数、堆垛方式和单双面反应对石墨烯化学反应行 为的影响. 本文将以这些成果为具体实例, 系统阐述我 们在石墨烯的光化学工程中的研究实践.

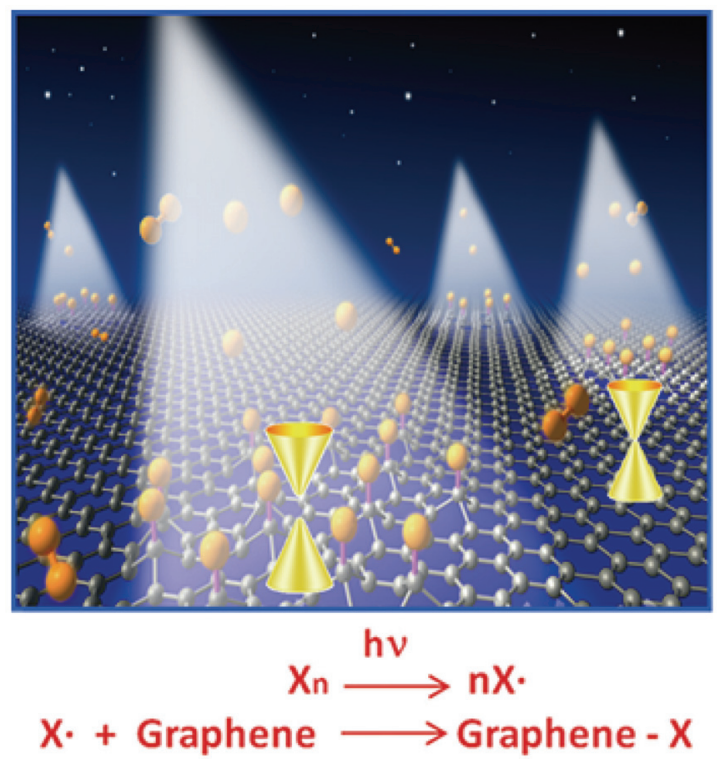

图 1 石墨烯的光化学自由基反应示意图

Figure 1 Schematic illustration of photo-induced free radicals-based photochemistry of graphene

\section{2 石墨烯的光氯化加成反应}

选择反应活性高的自由基是实现共价反应的关键. 为推断各类自由基与石墨烯反应的难易程度, 我们参考 了各自由基与氢原子间(R-H)的键解离能 ${ }^{[72]}$ 大小笁选高 活性自由基, 并通过光引发生成自由基. 键解离能越高, 则生成的自由基越不稳定, 夺氢能力越强, 通常表现为 反应活性越高 ${ }^{[73]}$. 自由基的夺氢能力排序如下:

\section{$\mathrm{F} \bullet>\mathrm{HO} \bullet>\mathrm{F}_{3} \mathrm{C} \bullet>\mathrm{H} \bullet, \mathrm{Cl} \bullet, \mathrm{MeO} \bullet>\mathrm{Ph} \bullet>\mathrm{Me} \bullet>\mathrm{Et} \bullet>$ $\mathrm{Cl}_{3} \mathrm{C} \bullet, \mathrm{Br} \bullet>\mathrm{PhCH}_{2} \bullet>\mathrm{Ph}_{3} \mathrm{C} \bullet>\mathrm{I} \bullet$}

目前已报导的石墨烯自由基反应涉及到的 $\mathrm{H} \bullet \mathrm{F} \bullet$ 、 $\mathrm{Ph} \cdot \mathrm{HO} \cdot$ 均为夺氢能力强的自由基. 氯自由基是最常见 的光生自由基, 可通过光解氯气分子产生, 易与苯发生 加成反应. 著名的农药六六六一一六氯环已烷即是利用 氯气在日光灯辐照下产生氯自由基, 与苯分子发生加成 反应制备得到的 ${ }^{[74]}$. 石墨烯可看作是脱氢的苯环在二
维空间无限延伸而成. 受该反应启发，我们发展了石墨 烯的光氯化加成反应，并研究了反应的机理及氯化石墨 烯的性质.

氯分子对 $250 \sim 450 \mathrm{~nm}$ 波长的光均有吸收，其最大 吸收峰位于 $330 \mathrm{~nm}$ 附近. 因此选用发光波段为 $200 \sim$ $1100 \mathrm{~nm}$ 的氙灯作为光源，并滤掉长波长的光，以避免 其带来的热效应. 氙灯光照下氯气分子解离成高活性的 氯自由基(图 2a), 通过自由基加成反应键连到石墨烯上, 实现了光氯化修饰. 光氯化反应可以在短短几分钟内实 现石墨烯的高效氯修饰. 反应后拉曼谱图上产生了强烈 的 D 峰, X 射线光电子能谱(XPS)图中新出现了来源于 $\mathrm{C}-\mathrm{Cl}$ 键的强峰, 说明 $\mathrm{Cl}$ 与石墨烯之间形成了共价键 (图 2b). 反应后石墨烯骨架结构仍旧保持, 高度比本征 石墨烯略高. 电阻显著增加约四个数量级, 且表现出明 显的 $\mathrm{p}$ 掺杂. 变温电学测试结果表明, 单层石墨烯经氯 化反应后打开了约 $45 \mathrm{meV}$ 的带隙(图 2c). 由于光氯化 反应的均匀性，利用抗氯掩模板或光掩模，很容易实现 石墨烯的图案化修饰. 如图 2d 所示, D 峰峰强 mapping 图上均匀的绿色表明氯化区域修饰程度非常均匀, 而黑 色区域仍为本征石墨烯. 颜色均匀的网格图形说明实现 了均匀的、图案化的光氯化修饰. 光氯化反应具有高效、 清洁、均匀、易于图案化及不破坏石墨烯结构的特点, 它 为构建石墨烯基功能材料和电子学器件提供了新的思 路, 通过对引入的 $\mathrm{C}-\mathrm{Cl}$ 键转化也为石墨烯进一步功能 化提供了新方法 ${ }^{[46]}$.

我们也通过拉曼、电学测试和密度泛函理论计算研 究了石墨烯光氯化反应的过程. 光氯化反应初期, 氯自 由基倾向于在石墨烯表面随机分布，电子从石墨烯向氯 自由基转移，但并未成键. 此时的石墨烯发生了严重 $\mathrm{p}$ 掺杂, 骨架结构并未改变, 我们称之为氯一石墨烯电荷 转移复合物. 随着反应时间延长，氯自由基在石墨烯表 面吸附密度增大，电荷转移复合物可能会转变成两种稳 定态之一：一种是碳氯共价成键态．若两个氯原子刚好 在石墨烯六元环的对位同时出现，则会形成两个碳-氯 键. 氯倾向于以六元环的形式聚集，单面的最大覆盖度 不超过 $25 \%$. 另一种稳定态是非键态. 当氯原子处在石 墨烯六元环的邻位时, 相邻氯原子会复合成氯分子并从 石墨烯表面脱附(图 3a) ${ }^{[75]}$.

实验跟踪光氯化过程发现，反应初期存在氯自由基 积累的阶段. 氯自由基的积累是反应的决速步，它决定 了氯化反应的速率是先慢后快的. 经过短时间光氯化处 理后，石墨烯并未与氯自由基成键，但表现出显著的 $\mathrm{p}$ 掺杂和电导增大效应(图 3b), 推测此时形成的是氯一石 墨烯电荷转移复合物. 电荷转移复合物的形成增加了石 墨烯电导，调节了石墨烯的带隙，可望用于透明导电薄 膜和 FET 器件 ${ }^{[76]}$. 
(a)

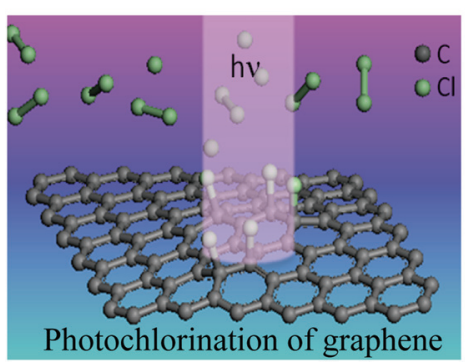

(c)

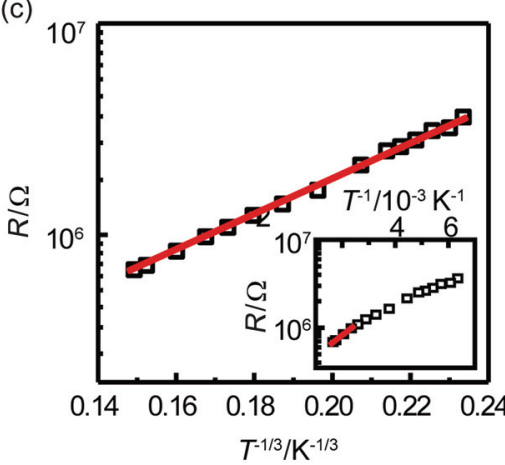

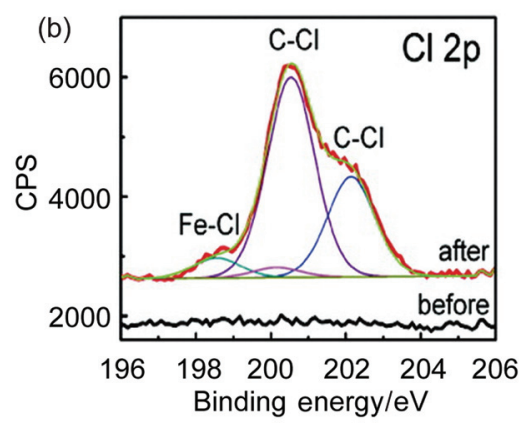

(d)

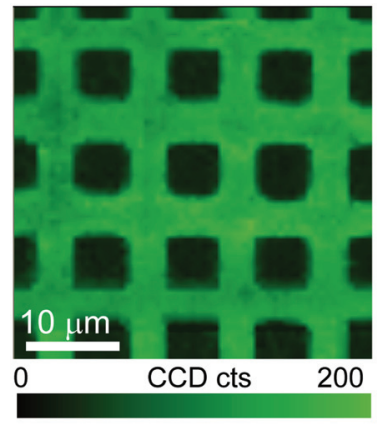

图 2 (a)石墨烯光氯化加成反应示意图. (b)光氯化反应前(黑)后(红)石墨烯的 XPS Cl 2p 高分辨分谱图. (c)氯化石墨烯的变温测试结果: 电阻 $R$ 与 $T^{-1 / 3}$ 的半对数图. 插图: 电阻 $R$ 与 $T^{-1}$ 的半对数图. (d)CVD 生长石墨烯图案化光氯化修饰后的拉曼 $\mathrm{D}$ 峰峰强的二维平面图 ${ }^{[46]}$

Figure 2 (a) Scheme of the photochemical chlorination of graphene. (b) High resolution XPS Cl 2p spectra of graphene before (black curve) and after photochlorination (red curve). (c) Variable-temperature electrical results of photochlorinated graphene. Semilog plot of the resistance $R$ vs $T^{-1 / 3}$ of the chlorinated graphene. Inset: Semilog plot of $R$ vs $T^{-1}$. (d) D band mapping for a CVD-grown graphene sample after a patterned photochlorination

(a)

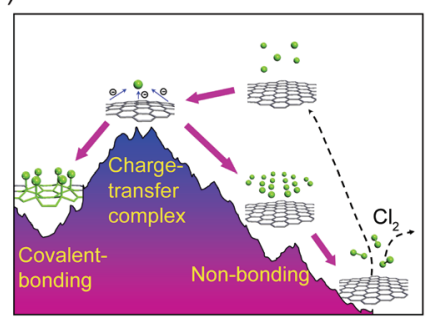

(b)

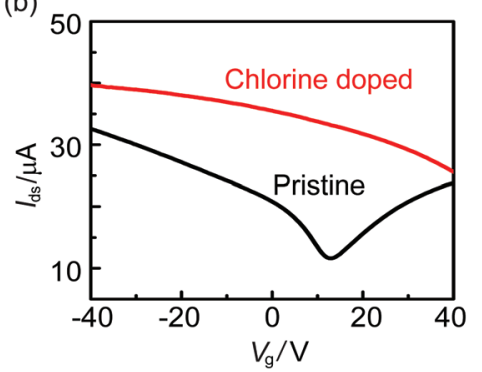

图 3 (a)石墨烯光氯化反应过程中各吸附构象演变的示意图 ${ }^{[75]}$. (b)石墨烯器件经 $2 \mathrm{~min}$ 光氯化处理前(黑)后(红)的 $I_{\mathrm{ds}}-V_{\mathrm{g}}$ 图(源漏偏压: 100 mV) ${ }^{[76]}$ Figure 3 (a) Schematic representation of the evolution of various adsorption configurations during chlorination of graphene. (b) $I_{\mathrm{ds}}-V_{\mathrm{g}}$ characteristics of a single-layer graphene device before (black curve) and after $2 \mathrm{~min}$ chlorine doping (red curve) at source drain bias of $100 \mathrm{mV}$

\section{3 石墨烯的光甲基化加成反应}

有机过氧化物是一种常见的自由基引发剂, 在光 照 ${ }^{\left[{ }^{[7]}\right.}$ 以及加热 ${ }^{[78]}$ 等条件下可以产生高活性的自由基. 由于有机过氧化物的种类繁多, 且生成的自由基通常反 应活性较高, 因此利用有机过氧化物的自由基反应可在 石墨烯表面上修饰不同的基团, 调控石墨烯的表面性质 和电荷掺杂. Brus 小组 ${ }^{\left[{ }^{[9]}\right.}$ 利用光催化过氧化苯甲酰 (BPO)分解的方法, 实现了石墨烯的苯基化修饰. 我们 利用过氧化叔丁基醚(DTBP)作为自由基产生剂, 在温 和的条件下均裂产生自由基, 发展了石墨烯的甲基化修 饰方法 ${ }^{[80]}$.

反应原理如图 4a 所示, DTBP 在光照条件下均裂生 成氧叔丁基自由基后, 进一步裂解得到甲基自由基. 生
成的甲基自由基与石墨烯反应，得到甲基化的石墨烯. 反应后的石墨烯的红外光谱中在 2877 和 $2971 \mathrm{~cm}^{-1}$ 处出 现了两个新的甲基的 $\mathrm{C}-\mathrm{H}$ 振动峰 ${ }^{[1]}$, 表明反应后的石 墨烯上引入了甲基(图 4b). 实验发现, 石墨烯的甲基化 加成过程是可逆的, 且光照是过氧化物与石墨烯加成反 应顺利进行的必要条件.

经甲基化修饰后石墨烯的电阻增大，高度甲基化的 石墨烯表现出绝缘体的性质，其面电阻高达约 $10^{11}$ $\Omega / \mathrm{sq}$. 同时, 石墨烯器件的狄拉克点随着反应时间的增 加逐渐负移，这表明新引入的甲基对石墨烯具有 $\mathrm{n}$ 型掺 杂的效应. 作为一种实现石墨烯稳定 $\mathrm{n}$ 掺的方法, 石墨 烯的光甲基化加成反应在制备石墨烯场效应晶体管以 及石墨烯 pn 结的领域有着广阔的应用前景. 
(a)

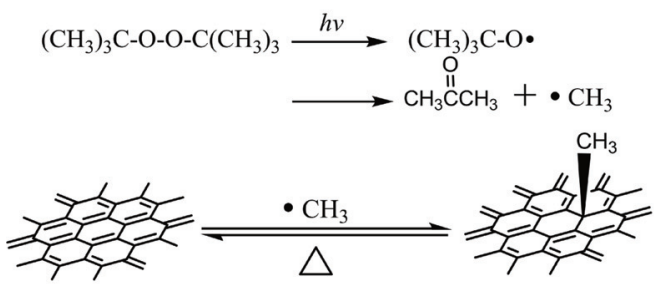

(b)

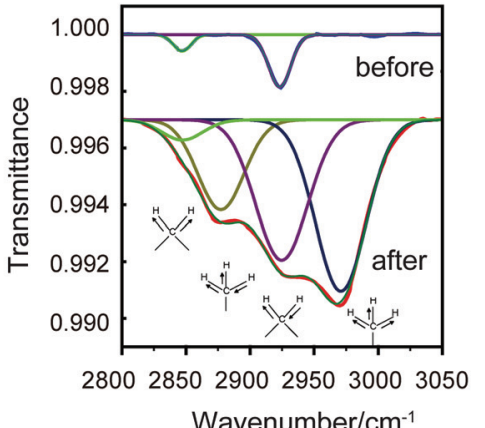

图 4 (a)石墨烯与 DTBP 反应的机理图. (b)甲基化前后石墨烯样品的红外吸收谱图 ${ }^{[80]}$

Figure 4 (a) Scheme of the reaction between di-tert-butyl peroxide and graphene. (b) IR spectra of pristine graphene (blue curve) and methylated graphene (red curve)

\section{4 石墨烯的光催化氧化反应}

光催化是一种重要的光化学反应现象, 它的实现依 赖于宽带隙半导体能够在紫外可见光下产生空穴-电子 对的分离, 其中光催化剂以对二氧化钛 $\left(\mathrm{TiO}_{2}\right)$ 的研究最 为广泛. 1972 年, Honda 和 Fujishima 在 $\mathrm{n}$ 型半导体 $\mathrm{TiO}_{2}$ 表面发现水的光电催化分解现象 ${ }^{[82]}$, 掀起了光催化的研 究热潮. $\mathrm{TiO}_{2}$ 是一种常见的 $\mathrm{n}$ 型半导体, 根据固体物理 理论, 半导体浸入溶液或置于空气中时表面电荷将重新 分布, 形成界面双电层, 表面电子能级发生弯曲出现边 缘能级并伴随形成有一定宽度的空间电荷层. 对于 $\mathrm{n}$ 型 $\mathrm{TiO}_{2}$ 而言, 边缘能级向上弯曲, 其位置与被吸附物氧化 还原电势的匹配程度, 决定了 $\mathrm{TiO}_{2}$ 光催化反应的能力. 热力学允许的光催化氧化还原反应, 要求受体电势比 $\mathrm{TiO}_{2}$ 的导带电势低(更正), 给体电势比 $\mathrm{TiO}_{2}$ 价带电势高 (更负). 在 $\mathrm{pH}$ 值为 7 的情况下, $\mathrm{TiO}_{2}$ 光生空穴的氧化电 势位置在 $+2.53 \mathrm{~V}$ (相对于标准氢电极, vs. SHE), 低于 $\cdot \mathrm{OH} / \mathrm{H}_{2} \mathrm{O}(+2.27 \mathrm{~V}$ vs. SHE $)$ 和 $\mathrm{H}_{2} \mathrm{O}_{2} / \mathrm{H}_{2} \mathrm{O}(+1.53 \mathrm{~V}$ vs. SHE)氧化还原对的电势位置, 因而空穴可以将 $\mathrm{H}_{2} \mathrm{O}$ 氧 化成为 $\cdot \mathrm{OH}$ 和 $\mathrm{H}_{2} \mathrm{O}_{2}$. 与此对应, $\mathrm{TiO}_{2}$ 导带电势为 -0.52 $\mathrm{V}\left(\mathrm{vs}\right.$. SHE), 其电子还原电势低于 $\mathrm{O}_{2} / \mathrm{O}_{2}{ }^{-{ }^{-}}(-0.28 \mathrm{~V}$ vs. SHE) 和 $\mathrm{O}_{2} / \mathrm{H}_{2} \mathrm{O}_{2}(+0.28 \mathrm{~V}$ vs. SHE) 氧化还原对的位置, 因而光生电子还可以将 $\mathrm{O}_{2}$ 还原得到 $\mathrm{O}_{2}{ }^{-}$和 $\mathrm{H}_{2} \mathrm{O}_{2}{ }^{[83,84]}$. 在不同的实验条件下, $\mathrm{TiO}_{2}$ 在紫外光激发下产生的含氧 基团, 例如 $\cdot \mathrm{OH} 、 \mathrm{H}_{2} \mathrm{O}_{2}$ 和 $\mathrm{O}_{2}{ }^{-}$等都可以作为活性中间体 实现后续的氧化反应 ${ }^{[55,86]}$. 根据上述光催化反应原理, 我们提出了石墨烯的光催化氧化修饰方法, 即利用 $\mathrm{TiO}_{2}$ 在紫外光下产生的空穴-电子对, 在催化剂表面分别与 周围空气中的 $\mathrm{H}_{2} \mathrm{O}$ 和 $\mathrm{O}_{2}$ 分子发生氧化还原反应, 产生 活性的含氧自由基进攻石墨烯, 实现光催化氧化修 饰 ${ }^{[67]}$.

如图 5 所示, 光催化氧化反应首先在石墨烯表面引 入羟基、环氧、羰基和羧基等含氧基团实现氧化修饰, 进 一步反应会导致碳骨架逐渐分解从而得到小分子气态 稳定产物, 如 $\mathrm{CO}$ 和 $\mathrm{CO}_{2}$ 等. 在氧化修饰的基础上将反 应时间延长, 最终将实现石墨烯的可控剪裁, 我们将这
种剪裁方法形象地称为“光催化剪纸技术”. 从反应原理 上讲, 光催化剪纸技术与其它石墨烯切割方法相比, 不 依赖于溶液相，避免了在石墨烯表面引入额外的污染 源, 在空气环境下借助光化学反应即可实现石墨烯的剪 裁, 并且剪裁图案依赖于模板的设计. 以偏析生长的石 墨烯为例, 利用条纹状 $\mathrm{TiO}_{2}$ 掩模板进行光催化氧化切 割, 即可得到石墨烯条纹, 条纹宽度取决于 $\mathrm{TiO}_{2}$ 掩模板 的设计(图 6a). 值得一提的是, 光催化剪裁方法对石墨 烯的担载基底没有要求，原则上任何与紫外光相兼容的 基底都可以用来承载石墨烯，其中包括很多透明柔性塑 料基底. 例如石墨烯可以在聚对苯二甲酸乙二酯(PET) 基底表面实现光催化切割(图 6b). 在柔性透明基底表面 实现石墨烯的光催化切割为制备柔性透明的全碳器件 提供了一条可行的思路.

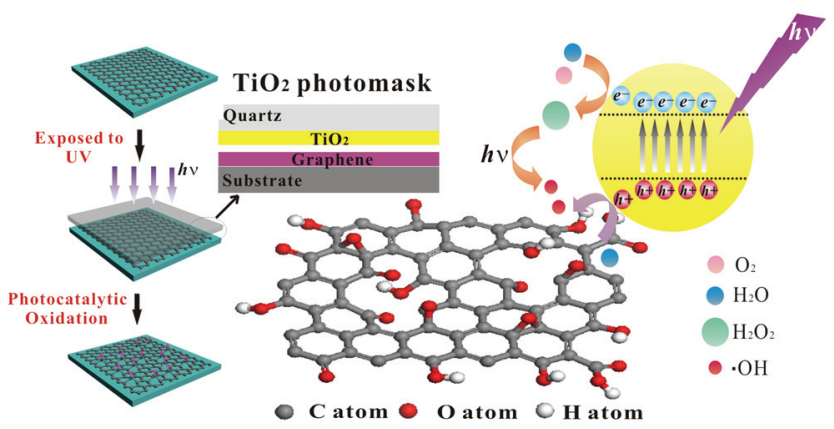

图 5 石墨烯的光催化氧化修饰原理示意图, 左侧为实验体系及流程 图 ${ }^{[67]}$

Figure 5 Schematic illustration of the photocatalytic approach to engineer graphene. Left panel is the experimental system and flow chart

石墨烯剪裁后出现的边缘电子态将影响石墨烯微 纳结构的电学性质, 边缘的散射效应也会急剧降低导电 沟道的迁移率 ${ }^{[35,87]}$. 在不破坏石墨烯表面完整性的前提 下对其实现 “虚拟” 切割则可以解决由边缘结构所引入 的上述问题. 石墨烯经过光催化氧化修饰之后首先形成 石墨烯氧化物结构，电学测量证实氧化物的面电阻可以 达到 $10^{9} \Omega / \mathrm{sq}$, 近似于绝缘态. 利用形成的石墨烯氧化 物作为隔离, 构筑石墨烯点阵结构可以实现对一片石墨 烯的 “虚拟” 切割. 如图 6c 所示, 亮色区域为反应后 
(a)

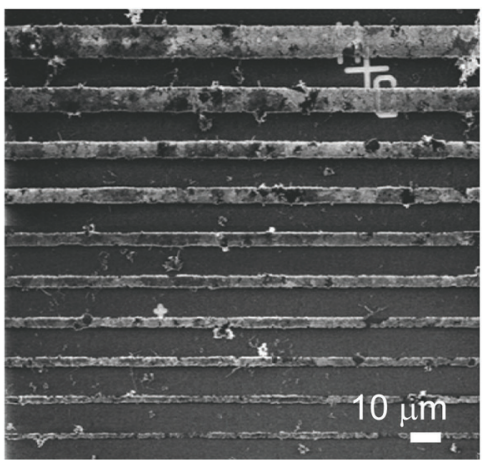

(b)

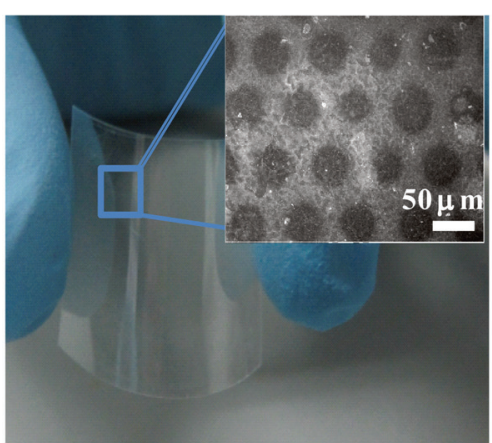

(c)
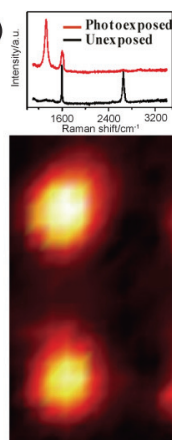

(d)

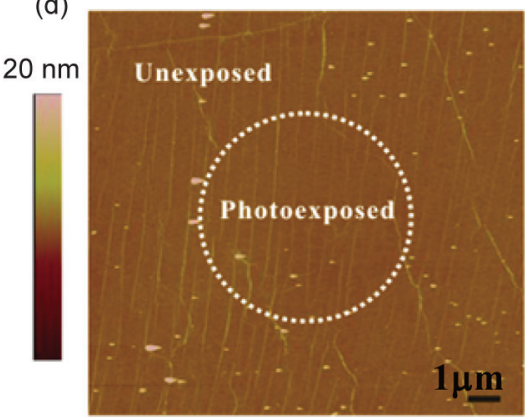

(e)

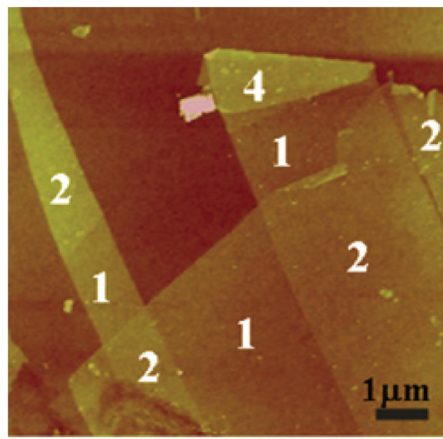

(f)

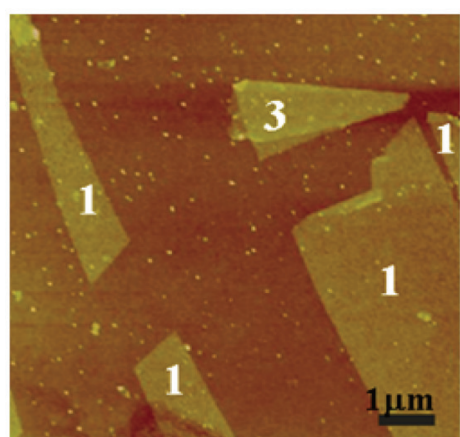

图 6 石墨烯的光催化氧化剪裁与逐层减薄. (a)偏析生长石墨烯的光催化切割, 利用光催化剪裁得到的不同宽度的石墨烯条带. (b)塑料基底表面 的石墨烯光催化切割. (c)石墨烯与石墨烯氧化物的超晶格结构，插图为对应区域的拉曼光谱. (d)石墨烯虚拟切割后表面的 AFM 图像，白色圆圈内 为形成的石墨烯氧化物区域. 多层石墨烯光催化氧化反应前(e)后(f)的形貌结构, 对应层数标注于图上 ${ }^{[67]}$

Figure 6 Photocatalytic patterning and layer-by-layer thinning of graphene. (a) SEM image of a series of segregated graphene ribbons show differences in width after photocatalytic patterning. (b) Photocatalytic patterning of graphene on plastic substrate. (c) D/G intensity ratio mapping of CVD-graphene after mild photocatalytic treatment using a circular $\mathrm{TiO}_{2}$ photomask. Inset shows the Raman spectra of photoexposed and unexposed areas. (d) AFM image of GO-patterned graphene, within the white circle is graphene oxide. AFM images of few-layer graphene before (e) and after (f) photocatalytic oxidation, respectively, in which the labeled numbers correspond to the layer thickness

的氧化石墨烯，暗色区域为本征石墨烯，表面氧化区域 的结构呈现与模板相对应的图案. 利用 AFM 表征反应 后的结构, 表面依然完整连续(图 6d), 证明局域反应并 没有破坏石墨烯的碳骨架，从而实现石墨烯的 “虚拟” 切割.

由于完美的石墨烯晶格结构具备强烈的化学惰性, 且多层石墨烯的层内与层间存在各向异性，我们发现在 石墨烯的光催化氧化反应过程中层内反应和层间反应 速率存在明显差异. 石墨烯晶畴边界存在较高的化学活 性，通常优先发生反应 ${ }^{[88,89]}$. 对于多层石墨烯，其层内 反应是一个加速过程, 层间反应则近乎匀速进行. 实验 发现, 在反应最初阶段, 层内与层间反应速率的比值为 9.7; 当反应 $2 \mathrm{~h}$ 后, 这一比值继续增大至 18.4. 基于此 我们利用光催化氧化反应实现了多层石墨烯的逐层减 薄. 采用机械剥离的方法制得多层共存的石墨烯, 如图 6e 和 f 所示, 光催化氧化反应前, 利用 AFM 表征石墨烯 的表面形貌(层数分布标注于图), 反应后再次对同一区 域的石墨烯成像. 通过对比发现, 一层石墨烯均匀地剥 离下来, 原来的单层区域已经完全消失. 通过测量, 石 墨烯的双层或三层区域高度降低了 $0.32 \sim 0.45 \mathrm{~nm}$, 恰 好对应一层石墨烯的高度分布 ${ }^{[90]}$.

\section{5 石墨烯自由基反应中的维度效应}

研究二维石墨烯分子的自由基反应行为对于理解 和调控石墨烯的化学反应尤为重要. 石墨烯在垂直于平 面的维度上的变化如层数、堆垛方式以及单面和双面的 差异赋予了石墨烯家族丰富多彩的性质. 光氯化具有高 效、均匀和对石墨烯结构无破坏性等特点，且由光引发 $\mathrm{Cl}-\mathrm{Cl}$ 键均裂产生的氯自由基是唯一的自由基物种 ${ }^{[46]}$. 因此, 我们利用光氯化反应作为探针, 研究了石墨烯作 为二维平面大分子所特有的层数、堆垛方式和单双面反 应效应，并实现了单层石墨烯的双面对称和非对称修 饰 ${ }^{[76]}$. 此外, 我们通过光甲基化反应证实了石墨烯的边 缘往往是化学反应中的活性位点 ${ }^{[80]}$.

\section{1 层数效应}

石墨烯的层数也会显著影响它的化学性质. 对于大 多数的反应来说, 如氧化反应 ${ }^{[68]}$ 和氢化反应 ${ }^{[65]}$, 层数越 多，石墨烯的反应活性越低. 通常认为不同层数的石墨 烯反应活性差异主要源自于共价反应过程中的反应势 垒差异 ${ }^{[65]}$. 与刚性的石墨不同，石墨烯有很多内在和外 部引起的起伏 ${ }^{[91]}$. 起伏带来的应力和弯曲会使 $\mathrm{C}-\mathrm{C}$ 键 形变, 共价修饰时会降低从 $\mathrm{sp}^{2}$ 到 $\mathrm{sp}^{3}$ 杂化结构转变引起 的形变势垒. 当层数增加时, 石墨烯片层变硬, 从 $\mathrm{sp}^{2}$ 到 
$\mathrm{sp}^{3}$ 转变的形变势垒增大, 导致石墨烯与自由基共价反 应变得困难. 我们的研究结果表明, 除了反应势垒的差 异外, 不同层数石墨烯在反应过程中的能带结构变化也 会影响石墨烯的化学反应活性.

光氯化反应后, 如图 7a 所示, 不同层数的石墨烯的 拉曼谱图上均出现了缺陷峰 D 峰. 对于单层区域, $2 \mathrm{D}$ 峰 完全消失, $\mathrm{D}$ 和 $\mathrm{G}$ 峰展宽严重, 说明氯自由基反应引入 了高密度的缺陷. 而 $\mathrm{AB}$ 堆垛双层石墨烯的 $I_{\mathrm{D}} / I_{\mathrm{G}}$ 比值最 小, 说明光氯化反应引入的缺陷密度最低. 依据不同层 数的石墨烯在相同氯化反应条件下反应后的拉曼结果, 我们推断石墨烯在光氯化反应中的反应活性顺序为: $1 \mathrm{~L}$ $>3 \mathrm{~L}>4 \mathrm{~L}>>2 \mathrm{~L}$. 对不同层数石墨烯光氯化反应后的 拉曼 $I_{\mathrm{D}} / I_{\mathrm{G}}$ 比值统计发现(图 7b 中蓝线), $\mathrm{AB}$ 堆垛双层的 $I_{\mathrm{D}} / I_{\mathrm{G}}$ 比值显著低于其它层数, 说明双层的氯化修饰程度 低, 进一步证实了光氯化中双层石墨烯的反应情性. 而 迄今报导的绝大多数石墨烯反应, 如氧化反应 ${ }^{[68]}$ 、氢化 反应 ${ }^{[65]}$ 、重氮盐反应 ${ }^{[22]}$ 等, 均是石墨烯的反应活性随着 层数增加而减小. 即使是在同为光引发的自由基反 应一一石墨烯与过氧化苯甲酰的反应中, 层数越多的石 墨烯, 其反应活性越低(图 7b 中红线). 此现象部分排除 了光照导致双层石墨烯反应惰性的可能. 依据前线分子 轨道理论, 石墨烯的最高占据轨道与氯原子的半占据轨 道的能级间距会显著影响轨道间的相互作用, 进而影响 氯与石墨烯间的化学反应. 对于双层石墨烯, 氯的强 $\mathrm{p}$ 掺杂作用以及不对称掺杂导致的带隙打开使得石墨烯

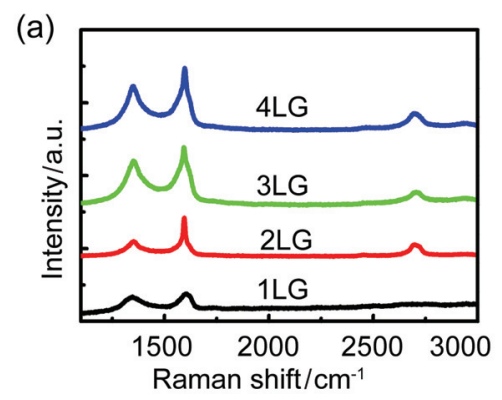

(c)

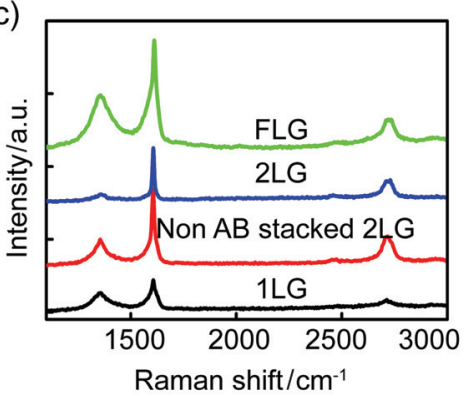

的最高占据轨道与氯原子的半占据轨道之间的能级间 隔增大，同时最高占据轨道附近的态密度降低，因而反 应活性较低. 双层石墨烯反常的化学惰性可能是层数增 加导致的反应形变势垒增加, 以及氯自由基反应过程中 石墨烯的能级结构变化共同作用的结果 ${ }^{[76]}$.

\section{2 堆垛效应}

二维的石墨烯大分子在垂直于平面的维度上存在 两个可变的参量, 层数和层与层之间的相对位置取向. 堆垛方式决定了层与层间的相互作用，进而对石墨烯的 诸多性质如能带结构 ${ }^{[93]}$ 、光学性质 ${ }^{[94]}$ 等产生显著影响. 在少层石墨烯中, 堆垛方式提供了一个重要但目前鲜有 研究的物理量来调控石墨烯的性质. 研究表明, 除了层 数外, 堆垛方式以及相应的层间耦合作用也会影响少层 石墨烯的能带结构和物理性质 ${ }^{[94,95]}$. 例如, 乱垛(非 $\mathrm{AB}$ 堆垛)的双层石墨烯在低能量处的色散关系是线性的, 与单层石墨烯类似，但费米速度显著低于单层石墨 烯 ${ }^{[95]}$. 当施加垂直非对称场时，无法打开乱垛双层石墨 烯的带隙. ABA 堆垛的三层石墨烯是半金属性质，导带 价带的能级交叠程度受垂直电场的调制，门电压越大， 导带价带交叠越多 ${ }^{[96]}$. 而 $\mathrm{ABC}$ 堆垛的三层与 $\mathrm{AB}$ 堆垛的 双层类似, 通过施加外电场可以引入可调的带隙 ${ }^{[97,98]}$.

目前对堆垛效应的研究主要集中在堆垛方式对石 墨烯物理性质的影响, 而堆垛与石墨烯化学性质的关系 是科学家们尚未涉足的领域. 我们借助简单、高效的光 氯化自由基反应探针，首次发现堆垛方式会影响石墨烯

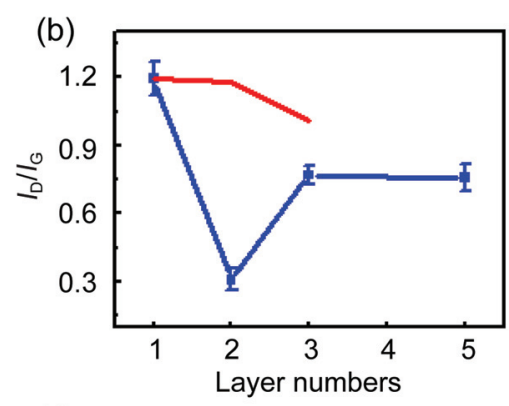

(d)

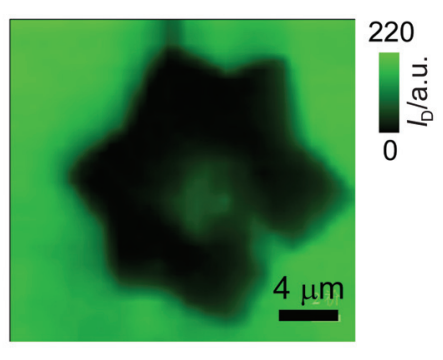

图 7 (a)石墨烯薄片上的单层石墨烯(1LG)、双层石墨烯(2LG)、三层石墨烯(3LG)和四层石墨烯(4LG)光氯化反应后的拉曼图谱. (b)光氯化(蓝)和 光苯基化(红)反应后石墨烯的 $I_{\mathrm{D}} / I_{\mathrm{G}}$ 比值与石墨烯层数的关系. (c)不同层数和堆垛方式的石墨烯样品光氯化反应后的拉曼光谱. 1LG、2LG、Non AB stacked 2LG、FLG 分别代表一层、 $\mathrm{AB}$ 堆垛双层、非 AB 堆垛双层石墨烯和少层石墨烯. (d)铜上 CVD 生长的石墨烯经光氯化反应后的拉曼 D 峰 峰强的二维平面图 ${ }^{[76]}$

Figure 7 (a) Raman spectra of single-layer (1LG), bilayer (2LG), tri-layer (3LG) and quadruple-layer (4LG) graphene presented in the same flake after photochlorination. (b) Statistical analysis of the intensity ratio of $\mathrm{D}$ band to $\mathrm{G}$ band $\left(I_{\mathrm{D}} / I_{\mathrm{G}}\right)$ as a function of layer numbers in the photochlorination (blue line) and the reaction with benzoyl peroxide (red line). (c) Raman spectra of the graphene sample after photochlorination. 1LG denotes single-layer graphene, 2LG denotes AB stacked bilayer graphene, Non AB-stacked 2LG denotes non-AB-stacked bilayer graphene, FLG denotes few-layer graphene. (d) Raman map of $\mathrm{D}$ band intensity for the CVD graphene grown on $\mathrm{Cu}$ after photochlorination 
的化学性质 ${ }^{[76]}$. 光氯化反应后, $\mathrm{AB}$ 堆垛的双层石墨烯拉 曼谱图上出现了微弱的 D 峰, 说明其反应程度低, 表现 出反应惰性. 同样是双层, 非 $\mathrm{AB}$ 堆垛的石墨烯反应后 $\mathrm{D}$ 峰强得多, 对应的 $I_{\mathrm{D}} / I_{\mathrm{G}}$ 比值也较大(图 7c). 同一区域 由 $\mathrm{AB}$ 堆垛双层折叠得到的非 $\mathrm{AB}$ 堆垛四层石墨烯与 $\mathrm{AB}$ 堆垛双层石墨烯氯化反应后的拉曼图谱类似, 说明氯化 反应中两者反应活性接近. 因此, 石墨烯的堆垛方式会 显著地影响石墨烯在自由基反应中的反应行为，只有 $\mathrm{AB}$ 堆垛的双层在氯化反应中表现出特殊的反应惰性.

石墨烯光氯化反应可用来快速、大面积显影石墨烯 的堆垛方式, 并实现选择性的化学修饰. 对于偏析生长 的非 $\mathrm{AB}$ 堆垛的石墨烯 ${ }^{[99]}$, 经氯化反应后, 不同层数的 石墨烯表现出相似的氯修饰效率. 对于铜上生长的 $\mathrm{AB}$ 堆垛的石墨烯 ${ }^{[31]}$, 可以通过微区拉曼二维扫描氯化后的 石墨烯来显影其堆垛方式. 双层区域的 D 峰峰强二维图 显示出均一的黑色(图 7d), 体现了 $\mathrm{AB}$ 堆垛双层石墨烯 均一的低反应活性. 单层石墨烯氯修饰效率高, 少层石 墨烯修饰效率较低，而双层石墨烯基本未修饰，实现了 氯在不同层数石墨烯上的选择性修饰.

\section{3 单双面自由基反应和双面非对称修饰}

单层石墨烯为开放的平面大分子, 所有碳原子均是 表面原子. 自由基物种可以从石墨烯的两面进攻与石墨 烯共价键合, 并可能形成确定化学计量比的石墨烯衍生 物. 石墨烯的不通透性使得反应物种无法接触到基底支
撑石墨烯的一侧，反应只在暴露的表面发生，因而是单 面反应. 为了使石墨烯上下表面均接触反应物, 实现双 面化学修饰, 需要将石墨烯县空以暴露下表面. 将石墨 烯的双表面暴露，反应物种能从两个方向进攻石墨烯， 可以用于研究双面与单面的反应差异、共价成键优先在 同一侧还是另一侧键合以及键合优先位点的位置、上下 两面修饰基团的相互作用等. 这些问题均是二维平面大 分子所独有的特点，也是石墨烯化学关心的问题.

实验中我们将机械剥离的石墨烯悬空在带凹槽的 硅片上(图 8a 插图)来研究单双面氯化反应. 光氯化反应 后，悬空区域和基底支撑区域单层石墨烯均出现 D 峰, 同时 $2 \mathrm{D}$ 峰衰减. 同一单层石墨烯悬空区域的 $I_{\mathrm{D}} / I_{\mathrm{G}}$ 值是 基底上对应比值的两倍多. 说明对于单层石墨烯双面氯 化的修饰效率大于两倍的单面氯化. 少层石墨烯双面氯 化的 $I_{\mathrm{D}} / I_{\mathrm{G}}$ 约是单面反应的 2 倍, 且随着层数增加, 单面 和双面氯化反应的 $I_{\mathrm{D}} / I_{\mathrm{G}}$ 值差异减小. 理论计算发现对于 单层石墨烯单面氯化，电荷转移复合物是能量优势 态 $^{[75]}$. 进一步形成对位的两个 $\mathrm{C}-\mathrm{Cl}$ 共价键需要克服 $0.5 \mathrm{eV}$ 的能垒. 而单层石墨烯双面氯化反应中, 氯原子 从石墨烯的两侧与相邻碳原子键合, 反应过程没有能 垒. 双面氯加成体系的总能量较低, 为 $-2.0 \mathrm{eV}$, 而单 面氯加成体系能量为 $-1.4 \mathrm{eV}$ (图 $8 \mathrm{~b}$ ). 这一结果表明双 面氯化形成 $\mathrm{C}-\mathrm{Cl}$ 共价键更容易, 且生成产物更稳定. 假设氯原子均匀化学吸附在单层石墨烯表面, 在相同氯 (a)

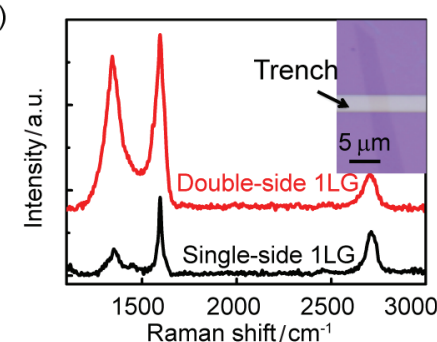

(d)

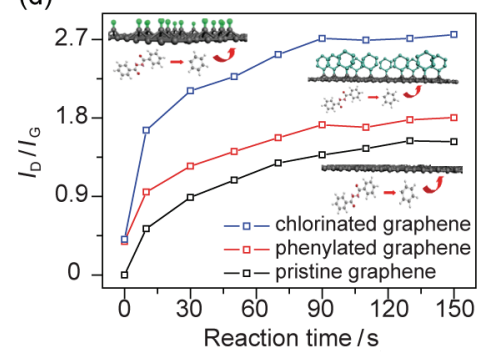

(b)

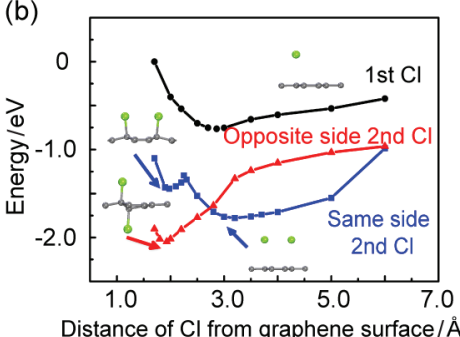

(e)

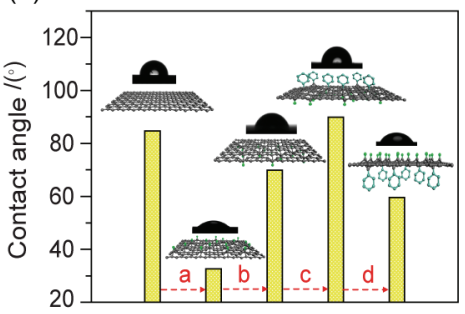

(c)

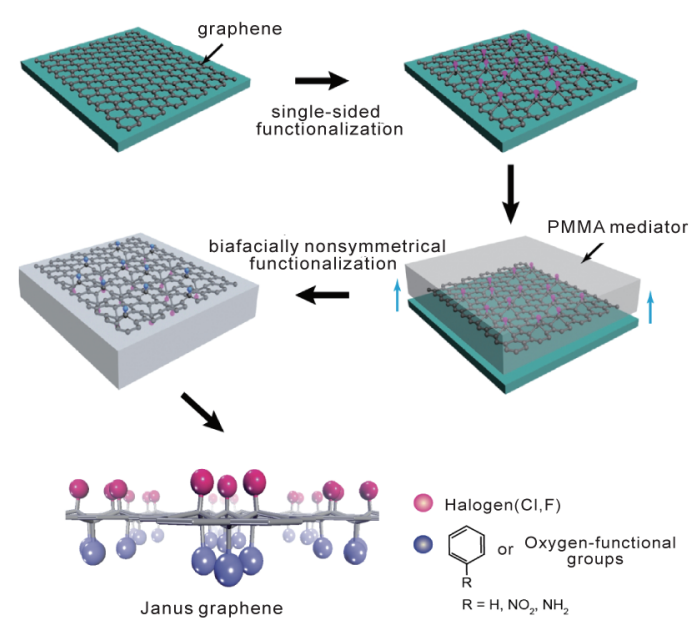

图 8 (a)单层石墨烯的悬空和基底支撑部分在氯化反应后的拉曼图谱. 插图为石墨烯在带凹槽的硅片的光学图片. 黄色区域为基底上的凹槽. (b) 单个氯原子吸附在石墨烯的一面(黑), 第二个氯原子吸附在同一面(蓝)和另一面(红)的势能曲线. 插图显示了对应的原子结构. (c)石墨烯双面非对 称修饰结构的制备流程示意图. (d)在原始、苯基化及氯化石墨烯的背面进行苯基化反应的动力学曲线. (e)单层石墨烯双面非对称修饰过程中表面 润湿性的变化 ${ }^{[76,101]}$

Figure 8 (a) Raman spectra of suspended and supported single-layer graphene after photochlorination. Inset shows the optical image of graphene suspended on $\mathrm{SiO}_{2} / \mathrm{Si}$ substrate with trenches. Yellow region represents a trench, on which graphene is free-standing. (b) Potential energy curves for a single $\mathrm{Cl}$ atom sticking to one side of graphene (black curve), and the second $\mathrm{Cl}$ atom sticking to the same side (blue curve) or the opposite side (red curve). Insets show sketches of the atomic structures. (c) Schematic illustration of the PMMA-assisted transfer procedure to synthesize Janus graphene. (d) Change of the $I_{\mathrm{D}} / I_{\mathrm{G}}$ in response to the phenylation time under different initial chlorination extent on the opposite side. (e) Static water contact angle measurements in the fabrication procedures of Janus graphene: single-sided photochlorination; exposure of graphene fresh side; single-sided phenylation; release of Janus graphene. The insets exhibit images of water droplets and schematic illustrations of the related surfaces 
覆盖度下, 双面氯加成结构比单面氯加成更加稳定. 实 验和理论计算结果均表明: 氯自由基更倾向于双面加 成. 当 $\mathrm{C}-\mathrm{Cl}$ 成键时, 下一步反应优先在石墨烯的另一 面上已有 $\mathrm{C}-\mathrm{Cl}$ 键的邻近 $\mathrm{C}$ 位置生成新的 $\mathrm{C}-\mathrm{Cl}$ 键. 单 层石墨烯的双面氯化反应活性比单面高, 可以达到更高 的氯覆盖度 ${ }^{[76]}$.

基底表面的石墨烯只能发生单面反应，受此启发若 将上下两个表面分别暴露于不同的化学环境, 将实现单 层石墨烯的双面非对称修饰. 计算表明，在基团的相同 覆盖度下, 相对于对称性修饰, 非对称修饰有利于打破 单层石墨烯的晶格对称性, 从而获得更大的理论带 隙 ${ }^{[100]}$; 同时该结构亦可以用于研究单层石墨烯两侧异 质基团在反应过程中能否透过中间单原子厚度的碳层 而交叉影响，即基团相互之间有无 “通话”。对于该问 题的理解和把握, 有助于我们在分子层面上深刻理解石 墨烯的非对称化学. 如图 8c 所示, 实验中我们采用聚甲 基丙烯酸甲酯(PMMA)薄膜作为操纵媒介, 利用光氯化 和光苯基加成反应分别修饰单层石墨烯的上下两面，首 次实现了单层石墨烯的双面共价非对称修饰, 并将这种 新颖的石墨烯衍生物结构命名为“Janus Graphene”[101]. 利用原位拉曼光谱监测石墨烯双面反应过程中的加成 速率, 我们发现, 苯基修饰和氯修饰的石墨烯在另一侧 的苯基化反应中表现出较大的活性差异. 如图 $8 \mathrm{~d}$ 所示, 我们选择初始 $I_{\mathrm{D}} / I_{\mathrm{G}}$ 几乎一致的两种单面修饰的石墨烯, 在相同条件下进行反面的苯基化反应. $10 \mathrm{~s}$ 反应时间内, 氯化石墨烯的 $I_{\mathrm{D}} / I_{\mathrm{G}}$ 极快增加, 证实背面的氯基团对于石 墨烯的活化能力强; 而苯基修饰的石墨烯对背面苯基化 反应的影响不大. 理论计算表明, 石墨烯在单面氯化过 程中修饰基团倾向于以六元环的形式向外铺展，氯原子 聚集形成团簇 ${ }^{[75]}$. 相反, 苯基化反应过程中, 苯环空间 位阻较大, 因此不能像氯原子那样在表面上形成密集的 团簇状 ${ }^{[102]}$. 在相同的 $I_{\mathrm{D}} / I_{\mathrm{G}}$ 下, 考虑到双面加成反应邻 位加成的优越性, 团簇状的氯原子由于引入了更多的活 化位点, 因而对石墨烯另一侧的反应带来较大的活化作 用, 零落分散的苯环则活化作用较弱.

化学修饰是进行石墨烯表面改性的有效手段, 通过 基团改性, 石墨烯表面将发生浸润性的变化. 我们细致 研究了单层石墨烯进行非对称修饰前后, 两侧接触角的 变化情况, 如图 8e 所示. 实验证实, 单层石墨烯两侧的 浸润性能够相互影响. 当石墨烯两侧分别键合疏水性 (如苯基)和亲水性(如 $\mathrm{Cl}$ )基团时, 最终结果将导致实际 接触角向中间方向靠拢，实验中测得上下两个表面最大 差异为 $30^{\circ}$, 该现象归因于单层石墨烯的厚度仅在 $1 \mathrm{~nm}$ 之内, 不足以完全隔绝下侧基团与水分子间的范德华 力. 单层石墨烯的双面非对称修饰具有重大意义. 从物 质合成角度来讲，丰富了石墨烯衍生物家族的“数据 库”, 同时提供了一类理想的模型结构, 研究石墨烯非 对称化学反应行为; 采用两种修饰方法同时对石墨烯进 行化学改性, 亦可以综合利用各自的优点, 弥补石墨烯
在实用化器件中的缺陷, 在生物化学传感中有着潜在的 应用。

\section{4 边缘效应}

石墨烯的边缘对于石墨烯的电学、磁学、光学以及 化学性质有着重要影响 ${ }^{[103,104]}$. 根据取向差异, 石墨烯 的边缘可以分为 zigzag 边和 armchair 边. Armchair 型的 石墨烯纳米带是一种半导体，其能级间隙随着纳米带的 宽度减小而不断增大; 相同尺寸的 zigzag 型石墨烯则是 一种半金属 ${ }^{[105]}$. 无序的石墨烯纳米带边缘会引入杂质 能级和散射位点, 降低其导电能力 ${ }^{[103]}$. 另外, 石墨烯的 边缘存在着较多的悬键和缺陷, 其化学性质更为活 泼 ${ }^{[92]}$. Zigzag 边由于存在着一对未成键电子, 相对于 armchair 边更容易与其他分子共价键合. 不同基团修饰 的石墨烯边缘对其性质也有显著影响. 羟基、羰基以及 酯基终止的石墨烯纳米带比碳氢键终止的石墨烯纳米 带的化学稳定性更高 ${ }^{[104]}$. 因此研究石墨烯的边缘反应, 对其边缘进行选择性的修饰是一种调控石墨烯性能和 构筑新型石墨烯器件的重要方法.

我们发现石墨烯的光甲基化反应是具有边缘优势 的. 当反应 $5 \mathrm{~min}$ 后, 边缘区域即可观察到很强的 D 峰, 而中央区域几乎没有 D 峰信号. 利用微区拉曼成像技术 监测石墨烯的甲基化过程发现，石墨烯的边缘区域相对 于中央区域 D 峰更强. 反应 $15 \mathrm{~min}$ 后, 边缘石墨烯的修 饰程度已接近饱和, 此时 Raman 光谱中 D 峰强度和 $G$ 峰强度的比值在 1.1 左右, 此时中央区域的石墨烯的 D 峰强度和 $\mathrm{G}$ 峰强度的比值仅为 0.3 左右. 这表明石墨
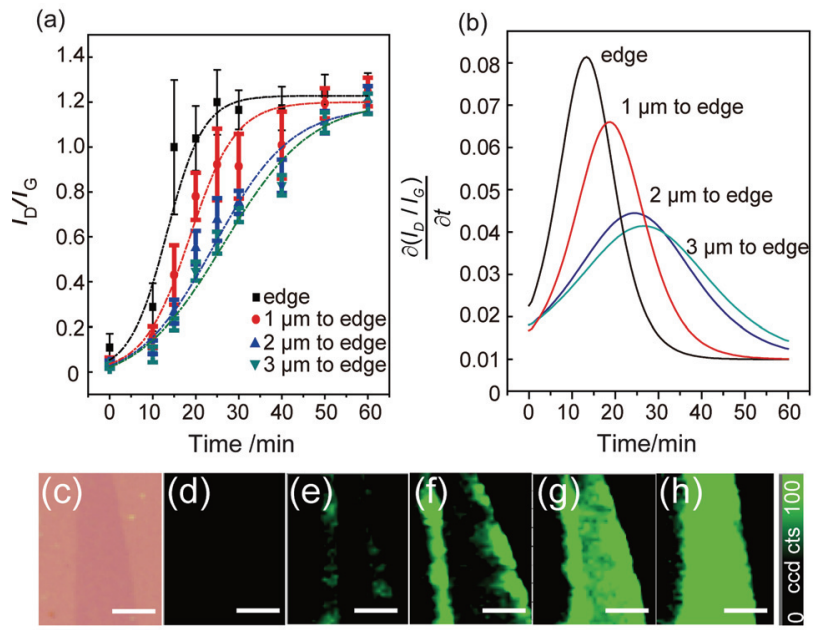

图 9 (a) 不同区域的石墨烯的 $\mathrm{D}$ 峰强度和 $\mathrm{G}$ 峰强度的比值随反应时 间的变化. (b)不同区域的石墨烯的 D 峰强度和 $\mathrm{G}$ 峰强度比值的导数随 反应时间的变化. (c)单层石墨烯的光学照片. $(\mathrm{d} \sim \mathrm{h})$ 分别为反应 $0 \mathrm{~min}$, $15 \mathrm{~min}, 30 \mathrm{~min}, 45 \mathrm{~min}$ 以及 $60 \mathrm{~min}$ 的石墨烯的拉曼 $\mathrm{D}$ 峰峰强的二维平 面图 ${ }^{[80]}$

Figure 9 (a) Time evolution of the $I_{\mathrm{D}} / I_{\mathrm{G}}$ for the spots on the single-layer graphene. (b) Derivatives of the intensity ratio shown in (a). (c) Optical image of the single-layer graphene. $(\mathrm{d} \sim \mathrm{h}) \mathrm{D}$ band mapping for the single-layer graphene shown in (c) after a series of reactions: (d) 0 min, (e) $15 \mathrm{~min}$, (f) $30 \mathrm{~min}$, (g) $45 \mathrm{~min}$, (h) $60 \mathrm{~min}$. The scale bars in (c $\sim \mathrm{h}$ ) are 5 $\mu \mathrm{m}$ 
烯的甲基化表现出明显的边缘优势. 另外, 通过观察 D 峰强度和 $\mathrm{G}$ 峰强度比值的导数随时间的变化, 我们发 现, 越接近边缘的区域导数值越大, 这表明, 越接近边 缘反应速度越快. 同时越接近边缘反应越容易到达饱 和. 随着反应的进行, 加成区域逐步向中央扩展, 最后 实现对石墨烯的均匀加成. 这一现象为石墨烯纳米带的 裁剪以及边缘可控修饰提供了一种新的思路 ${ }^{[80]}$.

\section{6 结论与展望}

本文系统阐述了我们在石墨烯共价化学修饰领域 的研究实践. 我们建立了一类石墨烯的光化学修饰方 法, 即利用光引发产生的高活性自由基来克服惰性石墨 烯的反应能垒，从而实现高效的石墨烯共价反应修饰. 这些光化学修饰方法包括: 光氯化加成反应、光甲基化 加成反应、光催化氧化反应、以及双面非对称加成反应 等. 光生自由基反应可以用作探针, 研究二维石墨烯大 分子的独特维度效应, 包括层数、堆垛方式、单双面和 边缘对石墨烯化学反应行为的影响. 单层石墨烯是最薄 的非通透膜, 这使得石墨烯的双面非对称修饰成为可 能，依此我们构筑了单分子层厚度的三明治结构 “Janus Graphene” . 石墨烯的光化学共价修饰具有高效可控、 反应多样以及易于图形化等优点，是实现石墨烯高效、 多元化修饰最有前景的方法之一.

石墨烯的共价化学是石墨烯研究领域的一个重要 分支, 在短短数年内已经取得长足的进步. 将经典的有 机反应用于石墨烯体系, 尤其借鉴碳纳米管化学和富勒 烯化学是人们的传统思维模式, 而根据石墨烯分子的特 殊性, 建立高效的化学修饰方法仍是石墨烯共价化学的 基本挑战. 自由基通常活性高, 能够有效克服石墨烯的 惰性，与石墨烯发生共价反应; 同时自由基种类繁多, 有很大的选择空间. 因此, 石墨烯的自由基反应化学将 是一个重要的发展方向.

共价化学修饰也为打开零带隙石墨烯的带隙提供 了可能, 这是制备新一代高性能石墨烯电子器件的基本 前提. 然而, 这种共价化学反应通常在石墨烯面内是随 机发生的, 难以实现原子水平的可控性, 当表面存在各 种高反应活性的缺陷态时, 此现象尤为严重. 所造成的 后果是化学修饰石墨烯的载流子迁移率和电导率严重 降低, 失去了本征石墨烯的优良特性. 周期性的化学修 饰, 即在石墨烯表面局部区域有选择性地引入化学官能 团, 形成周期性的马赛克结构有望解决这一难题 ${ }^{[100]}$. 我们建立的光化学修饰方法为马赛克石墨烯的制备提 供了非常有效的技术路径, 利用成熟的光掩模技术即可 实现，这方面的工作也有很大的探索空间.

最后, 石墨烯作为典型的二维原子晶体, 其共价化 学也可以部分延伸到其他二维原子晶体体系, 如 $\mathrm{MoS}_{2}$ 、 $h$-BN 以及 BNC 等石墨烯与其他二维原子晶体材料的杂 化体系. 此类二维材料的共价化学为研究化学反应的尺
寸效应提供了珍贵的模型体系，同时也为设计新型二维 材料提供了技术路线, 有望成为新的研究热点.

\section{作者简介}

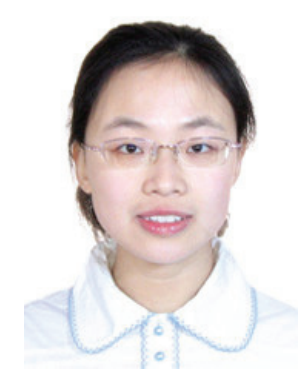

周琳, 1987 年生, 博士. 2008 年获得南京大学材料化学专 业理学学士学位. 2013 年在北京大学化学与分子工程学院获 得理学博士学位, 导师刘忠范院士. 主要从事石墨烯的化学修 饰研究.

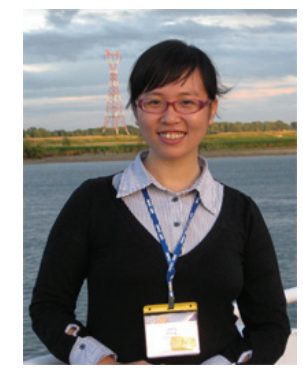

张黎明, 1985 年生, 博士. 2007 年获得山东大学化学专业 理学学士学位. 2012 年在北京大学化学与分子工程学院获得 理学博士学位, 导师刘忠范院士. 博士生阶段的主要研究工作 是发展了石墨烯的光催化氧化及单层石墨烯的非对称修饰方 法.

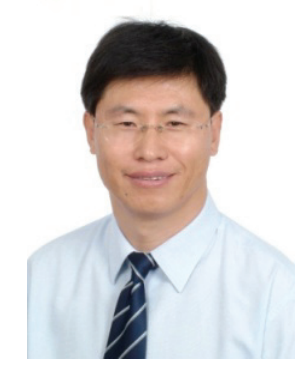

刘忠范, 北京大学化学与分子工程学院教授, 中国科学院 院士, 中组部万人计划杰出人才. 长期从事低维碳材料和纳米 化学研究, 在低维碳材料的光化学、CVD 生长以及能带工程 等研究方面取得重要成果, 发表学术论文 370 余篇, 获授权中 国发明专利 24 项。曾获国家自然科学二等奖、高等学校科学 技术奖自然科学一等奖、中国化学会一阿克苏诺贝尔化学奖以 及宝钢优秀教师特等奖等.

\section{References}

[1] Novoselov, K. S.; Geim, A. K.; Morozov, S. V.; Jiang, D.; Zhang, Y.; Dubonos, S. V.; Grigorieva, I. V.; Firsov, A. A. Science 2004, $306,666$.

[2] Landau, L. D. Phys. Z. Sowjetunion 1937, 11, 26.

[3] Peierls, R. E. Ann. I. H. Poincare1935, 5, 177.

[4] Mayorov, A. S.; Gorbachev, R. V.; Morozov, S. V.; Britnell, L.; 
Jalil, R.; Ponomarenko, L. A.; Blake, P.; Novoselov, K. S.; Watanabe, K.; Taniguchi, T.; Geim, A. K. Nano Lett. 2011, 11, 2396.

[5] Morozov, S. V.; Novoselov, K. S.; Katsnelson, M. I.; Schedin, F.; Elias, D. C.; Jaszczak, J. A.; Geim, A. K. Phys. Rev. Lett. 2008, 100, 016602.

[6] Novoselov, K. S.; Falko, V. I.; Colombo, L.; Gellert, P. R.; Schwab, M. G.; Kim, K. Nature 2012, 490, 192.

[7] Moser, J.; Barreiro, A.; Bachtold, A. Appl. Phys. Lett. 2007, 91, 163513.

[8] Balandin, A. A. Nat. Mater. 2011, 10, 569.

[9] Lee, C. G.; Wei, X. D.; Kysar, J. W.; Hone, J. Science 2008, 321, 385-388.

[10] Liu, F.; Ming, P. B.; Li, J. Phys. Rev. B 2007, 76, 064120.

[11] Nair, R. R.; Blake, P.; Grigorenko, A. N.; Novoselov, K. S.; Booth, T. J.; Stauber, T.; Peres, N. M. R.; Geim, A. K. Science 2008, 320, 1308 .

[12] Bunch, J. S.; Verbridge, S. S.; Alden, J. S.; van der Zande, A. M.; Parpia, J. M.; Craighead, H. G.; McEuen, P. L. Nano Lett. 2008, 8, 2458.

[13] Wu, Y. Q.; Lin, Y. M.; Bol, A. A.; Jenkins, K. A.; Xia, F. N.; Farmer, D. B.; Zhu, Y.; Avouris, P. Nature 2011, 472, 74.

[14] Gabor, N. M.; Song, J. C. W.; Ma, Q.; Nair, N. L.; Taychatanapat, T.; Watanabe, K.; Taniguchi, T.; Levitov, L. S.; Jarillo-Herrero, P. Science 2011, 334, 648.

[15] Schedin, F.; Geim, A. K.; Morozov, S. V.; Hill, E. W.; Blake, P.; Katsnelson, M. I.; Novoselov, K. S. Nat. Mater. 2007, 6, 652.

[16] Bae, S.; Kim, H.; Lee, Y. B.; Xu, X. F.; Park, J.-S.; Zheng, Y.; Balakrishnan, J.; Lei, T.; Kim, H. R.; Song, Y. I.; Kim, Y.-J.; Kim, K. S.; Özyilmaz, B.; Ahn, J.-H.; Hong, B. H.; Iijima, S. Nat. Nanotechnol. 2010, 5, 574.

[17] Novoselov, K. S.; Jiang, D.; Schedin, F.; Booth, T. J.; Khotkevich, V. V.; Morozov, S. V.; Geim, A. K. Proc. Natl. Avad. Sci. U. S. A. 2005, 102, 10451.

[18] Li, X. L.; Zhang, G. Y.; Bai, X. D.; Sun, X. M.; Wang, X. R.; Wang, E. G.; Dai, H. J. Nat. Nanotechnol. 2008, 3, 538.

[19] Shih, C. J.; Vijayaraghavan, A.; Krishnan, R.; Sharma, R.; Han, J. H.; Ham, M. H.; Jin, Z.; Lin, S. C.; Paulus, G. L. C.; Reuel, N. F.; Wang, Q. H.; Blankschtein, D. ; Strano, M. S. Nat. Nanotechnol. 2011, 6, 439.

[20] Stankovich, S.; Dikin, D. A.; Piner, R. D.; Kohlhaas, K. A.; Kleinhammes, A.; Jia, Y. Y.; Wu, Y.; Nguyen, S. T.; Ruoff, R. S. Carbon 2007, 45, 1558.

[21] Stankovich, S.; Dikin, D. A.; Dommett, G. H. B.; Kohlhaas, K. M.; Zimney, E. J.; Stach, E. A.; Piner, R. D.; Nguyen, S. T.; Ruoff, R. S. Nature 2006, 442, 282.

[22] Berger, C.; Song, Z. M.; Li, T. B.; Li, X. B.; Ogbazghi, A. Y.; Feng, R.; Dai, Z. T.; Marchenkov, A. N.; Conrad, E. H.; First, P. N.; de Heer, W. A. J. Phys. Chem. B 2004, 108, 19912.

[23] Zhang, C. H.; Fu, L.; Zhang, Y. F.; Liu, Z. F. Acta Chim. Sinica 2013, 71, 308. (张朝华, 付砧, 张艳锋, 刘忠范, 化学学报, 2013, 71, 308.)

[24] Cai, J. M.; Ruffieux, P.; Jaafar, R.; Bieri, M.; Braun, T.; Blankenburg, S.; Muoth, M.; Seitsonen, A. P.; Saleh, M.; Feng, X. L.; Mullen, K.; Fasel, R. Nature 2010, 466, 470.

[25] Reina, A.; Jia, X. T.; Ho, J.; Nezich, D.; Son, H. B.; Bulovic, V.; Dresselhaus, M. S.; Kong, J. Nano Lett. 2009, 9, 30.

[26] Li, X. S.; Cai, W. W.; An, J. H.; Kim, S.; Nah, J.; Yang, D. X.; Piner, R.; Velamakanni, A.; Jung, I.; Tutuc, E.; Banerjee, S. K.; Colombo, L.; Ruoff, R. S. Science 2009, 324, 1312.

[27] Zou, Z. Y.; Dai, B. Y.; Liu, Z. F. Sci. China-Chem. 2013, 43, 1. (邹 志宇, 戴博雅, 刘忠范, 中国科学: 化学, 2013, 43, 1.)

[28] Zhang, Y. F.; Gao, T.; Zhang, Y.; Liu, Z. F. Acta Phys-Chim. Sin. 2012，28，2456. (张艳锋, 高腾, 张玉, 刘忠范, 物理化学学报, 2012, 28, 2456.)

[29] Liao, L.; Bai, J. W.; Cheng, R.; Zhou, H. L.; Liu, L. X.; Liu, Y.; Huang, Y.; Duan, X. F. Nano Lett. 2011, 2653.

[30] Li, X. S.; Magnuson, C. W.; Venugopal, A.; Tromp, R. M.; Hannon, J. B.; Vogel, E. M.; Colombo, L.; Ruoff, R. S. J. Am. Chem. Soc. 2011, 133, 2816

[31] Yan, K.; Peng, H. L.; Zhou, Y.; Li, H.; Liu, Z. F. Nano Lett. 2011, 11,1106

[32] Dai, B. Y.; Fu, L.; Zou, Z. Y.; Wang, M.; Xu, H. T.; Wang, S.; Liu, Z. F. Nat. Commun. 2011, 2, 522.

[33] Yan, Z.; Lin, J.; Peng, Z. W.; Sun, Z. Z.; Zhu, Y.; Li, L.; Xiang, C. S.; Samuel, E. L.; Kittrell, C.; Tour, J. M. ACS Nano 2012, 6, 9110.
[34] Novoselov, K. S.; Geim, A. K.; Morozov, S. V.; Jiang, D.; Katsnelson, M. I.; Grigorieva, I. V.; Dubonos, S. V.; Firsov, A. A Nature 2005, 438, 197.

[35] Han, M. Y.; Özyilmaz, B.; Zhang, Y. B.; Kim, P. Phys. Rev. Lett. 2007, 98, 206805.

[36] Son, Y. W.; Cohen, M. L.; Louie, S. G. Nature 2006, 444, 347.

[37] Jiao, L. Y.; Zhang, L.; Wang, X. R.; Diankov, G.; Dai, H. J. Nature 2009, 458, 877 .

[38] Kosynkin, D. V.; Higginbotham, A. L.; Sinitskii, A.; Lomeda, J. R.; Dimiev, A.; Price, B. K.; Tour, J. M. Nature 2009, 458, 872.

[39] Bai, J. W.; Zhong, X.; Jiang, S.; Huang, Y.; Duan, X. F. Nat. Nanotechnol. 2010, 5, 190.

[40] Kim, M.; Safron, N. S.; Han, E.; Arnold, M. S.; Gopalan, P. Nano Lett. 2010, 10, 1125.

[41] Ohta, T.; Bostwick, A.; Seyller, T.; Horn, K.; Rotenberg, E. Science 2006, 313, 951 .

[42] Castro, E. V.; Novoselov, K. S.; Morozov, S. V.; Peres, N. M. R.; Lopes dos Santos, J. M. B.; Nilsson, J.; Guinea, F.; Geim, A. K.; Castro Neto, A. H. Phys. Rev. Lett. 2007, 99, 216802.

[43] Weitz, R. T.; Allen, M. T.; Feldman, B. E.; Martin, J.; Yacoby, A. Science 2010, 330, 812.

[44] Zhang, Y. B.; Tang, T. T.; Girit, C.; Hao, Z.; Martin, M. C.; Zettl, A.; Crommie, M. F.; Shen, Y. R.; Wang, F. Nature 2009, 459, 820.

[45] Feldman, B. E.; Martin, J.; Yacoby, A. Nat. Phys. 2009, 5, 889.

[46] Li, B.; Zhou, L.; Wu, D.; Peng, H. L.; Yan, K.; Zhou, Y.; Liu, Z. F. ACS Nano 2011, 5, 5957.

[47] Jeon, K. J.; Lee, Z.; Pollak, E.; Moreschini, L.; Bostwick, A.; Park, C. M.; Mendelsberg, R.; Radmilovic, V.; Kostecki, R.; Richardson, T. J.; Rotenberg, E. ACS Nano 2011, 5, 1042.

[48] Zhang, H.; Bekyarova, E.; Huang, J.-W.; Zhao, Z.; Bao, W. Z.; Wang, F. L.; Haddon, R. C.; Lau, C. N. Nano Lett. 2011, 11, 4047.

[49] Elias, D. C.; Nair, R. R.; Mohiuddin, T. M. G.; Morozov, S. V.; Blake, P.; Halsall, M. P.; Ferrari, A. C.; Boukhvalov, D. W.; Katsnelson, M. I.; Geim, A. K.; Novoselov, K. S. Science 2009, 323, 610.

[50] Nair, R. R.; Ren, W. C.; Jalil, R.; Riaz, I.; Kravets, V. G.; Britnell, L.; Blake, P.; Schedin, F.; Mayorov, A. S.; Yuan, S. J.; Katsnelson, M. I.; Cheng, H. M.; Strupinski, W.; Bulusheva, L. G.; Okotrub, A V.; Grigorieva, I. V.; Grigorenko, A. N.; Novoselov, K. S.; Geim, A. K. Small 2010, 6, 2877.

[51] Robinson, J. T.; Burgess, J. S.; Junkermeier, C. E.; Badescu, S. C.; Reinecke, T. L.; Perkins, F. K.; Zalalutdniov, M. K.; Baldwin, J. W.; Culbertson, J. C.; Sheehan, P. E.; Snow, E. S. Nano Lett. 2010, 10, 3001.

[52] Sun, Z. Z.; Pint, C. L.; Marcano, D. C.; Zhang, C. G.; Yao, J.; Ruan, G. D.; Yan, Z.; Zhu, Y.; Hauge, R. H.; Tour, J. M. Nat. Commun. 2011, 2, 559 .

[53] Li, D.; Muller, M. B.; Gilje, S.; Kaner, R. B.; Wallace, G. G. Nat. Nanotechnol. 2008, 3, 101.

[54] Lomeda, J. R.; Doyle, C. D.; Kosynkin, D. V.; Hwang, W. F.; Tour, J. M. J. Am. Chem. Soc. 2008, 130, 16201.

[55] Bonaccorso, F.; Sun, Z.; Hasan, T.; Ferrari, A. C. Nat. Photonics 2010, 4, 611 .

[56] Luo, Z. T.; Vora, P. M.; Mele, E. J.; Johnson, A. T. C.; Kikkawa, J. M. Appl. Phys. Lett. 2009, 94, 111909.

[57] Kim, J.; Cote, L. J.; Kim, F.; Huang, J. X. J. Am. Chem. Soc. 2009, $132,260$.

[58] Xia, Q. F.; Luo, D.; Li, Z. J. Acta Chim. Sinica 2012, 70, 2079. (夏 前芳, 罗丹, 李在均, 化学学报, 2012, 70, 2079.)

[59] Yang, S. B.; Feng, X. L.; Wang, X. C.; Müllen, K. Angew. Chem., Int. Ed. 2011, 50, 5339 .

[60] Zhang, Q.; Wu, S. Y.; He, M. W.; Zhang, L.; Liu, Y.; Li, J. H.; Song, X. M. Acta Chim. Sinica 2012, 70, 2213. (张谦, 吴抒遥, 何 茂伟, 张玲, 刘洋, 李景虹, 宋溪明, 化学学报, 2012, 70, 2213.)

[61] Xie, W. J.; Fu, Y. Y.; Ma, H.; Zhang, M.; Fan, L. Z. Acta Chim. Sinica 2012, 70, 2169. (谢文菁, 傅英懿, 马红, 张沫, 范楼珍, 化 学学报, 2012, 70, 2169.)

[62] Sun, X. M.; Liu, Z.; Welsher, K.; Robinson, J.; Goodwin, A.; Zaric, S.; Dai, H. J. Nano Res. 2008, 1, 203.

[63] Yang, K.; Zhang, S.; Zhang, G. X.; Sun, X. M.; Lee, S.-T.; Liu, Z. Nano Lett. 2010, 10, 3318.

[64] Kuila, T.; Bose, S.; Khanra, P.; Mishra, A. K.; Kim, N. H.; Lee, J. H. Biosens. Bioelectron. 2011, 26, 4637.

[65] Ryu, S.; Han, M. Y.; Maultzsch, J.; Heinz, T. F.; Kim, P.; Steigerwald, M. L.; Brus, L. E. Nano Lett. 2008, 8, 4597.

[66] Gong, P. W.; Wang, Z. F.; Li, Z. P.; Mi, Y. J.; Sun, J. F.; Niu, L. Y.; 
Wang, H. G.; Wang, J. Q.; Yang, S. R. RSC Adv. 2013, 3, 6327.

[67] Zhang, L. M.; Diao, S.; Nie, Y. F.; Yan, K.; Liu, N.; Dai, B. Y.; Xie, Q.; Reina, A.; Kong, J.; Liu, Z. F. J. Am. Chem. Soc. 2011, 133, 2706.

[68] Liu, L.; Ryu, S. M.; Tomasik, M. R.; Stolyarova, E.; Jung, N.; Hybertsen, M. S.; Steigerwald, M. L.; Brus, L. E.; Flynn, G. W. Nano Lett. 2008, 8, 1965.

[69] Bekyarova, E.; Itkis, M. E.; Ramesh, P.; Berger, C.; Sprinkle, M.; de Heer, W. A.; Haddon, R. C. J. Am. Chem. Soc. 2009, 131, 1336.

[70] Quintana, M.; Spyrou, K.; Grzelczak, M.; Browne, W. R.; Rudolf, P.; Prato, M. ACS Nano 2010, 4, 3527.

[71] Benson, S. W. J. Chem. Educ. 1965, 42, 502.

[72] Bansal, R. K. Organic Reaction Mechanisms, Tata McGraw Hill, New Delhi, 1978, Chapter 6.

[73] Mu, G. Z. Free Radical Reactions, Higher Education Press, Beijing, 1983, pp. $27 \sim 37$. (穆光照, 自由基反应, 高等教育出版社, 北京, 1983, pp. $27 \sim 37$.)

[74] Bowen, E. J.; Hinshelwood, C. N.; Sidgwick, N. V.; Thompson, H. W.; Wolfenden, J. H. Annu. Rep. Prog. Chem. 1932, $29,13$.

[75] Yang, M. M.; Zhou, L.; Wang, J. Y.; Liu, Z. F.; Liu, Z. R. J. Phys. Chem. C 2012, 116, 844.

[76] Zhou, L.; Zhou, L. S.; Yang, M. M.; Wu, D.; Liao, L.; Yan, K.; Xie, Q.; Liu, Z. R.; Peng, H. L.; Liu, Z. F. Small 2013, 9, 1388.

[77] Milas, N. A.; Surgenor, D. M. J. Am. Chem. Soc. 1946, 68, 205.

[78] Noyes, J. W. A.; Duncan, A. B. F.; Manning, W. M. J. Chem. Phys. 1934, 2, 717.

[79] Liu, H. T.; Ryu, S. M.; Chen, Z. Y.; Steigerwald, M. L.; Nuckolls, C.; Brus, L. E. J. Am. Chem. Soc. 2009, 131, 17099.

[80] Liao, L.; Song, Z. H.; Zhou, Y.; Wang, H.; Xie, Q.; Peng, H. L.; Liu, Z. F. Small 2013, 9, 1348.

[81] Bermudez, V. M.; Robinson, J. T. Langmuir 2011, 27, 11026.

[82] Fujishima, A.; Honda, K. Nature 1972, 238, 37.

[83] Mills, A.; Hunte, S. L. J. Photochem. Photobiol., A 1997, 108, 1.

[84] Fujishima, A.; Zhang, X. T.; Tryk, D. A. Surf. Sci. Rep. 2008, 63, 515.

[85] Ishibashi, K.-i.; Nosaka, Y.; Hashimoto, K.; Fujishima, A. J. Phys. Chem. B 1998, 102, 2117.

[86] Tatsuma, T.; Tachibana, S.-i.; Miwa, T.; Tryk, D. A.; Fujishima, A. J. Phys. Chem. B 1999, 103, 8033.

[87] Barone, V.; Hod, O.; Scuseria, G. E. Nano Lett. 2006, 6, 2748.
[88] Grantab, R.; Shenoy, V. B.; Ruoff, R. S. Science 2010, 330, 946.

[89] Huang, P. Y.; Ruiz-Vargas, C. S.; van der Zande, A. M.; Whitney, W. S.; Levendorf, M. P.; Kevek, J. W.; Garg, S.; Alden, J. S.; Hustedt, C. J.; Zhu, Y.; Park, J.; McEuen, P. L.; Muller, D. A Nature 2011, 469, 389.

[90] Geringer, V.; Liebmann, M.; Echtermeyer, T.; Runte, S.; Schmidt, M.; Ruckamp, R.; Lemme, M. C.; Morgenstern, M. Phys. Rev. Lett. 2009, 102, 076102 .

[91] Meyer, J. C.; Geim, A. K.; Katsnelson, M. I.; Novoselov, K. S.; Booth, T. J.; Roth, S. Nature 2007, 446, 60.

[92] Sharma, R.; Baik, J. H.; Perera, C. J.; Strano, M. S. Nano Lett. 2010 10,398 .

[93] Luican, A.; Li, G. H.; Reina, A.; Kong, J.; Nair, R. R.; Novoselov, K. S.; Geim, A. K.; Andrei, E. Y. Phys. Rev. Lett. 2011, 106, 126802.

[94] Wang, Y. Y.; Ni, Z. H.; Liu, L.; Liu, Y. H.; Cong, C. X.; Yu, T.; Wang, X. J.; Shen, D. Z.; Shen, Z. X. ACS Nano 2010, 4, 4074.

[95] Lopes dos Santos, J. M. B.; Peres, N. M. R.; Castro Neto, A. H. Phys. Rev. Lett. 2007, 99, 256802.

[96] Craciun, M. F.; Russo, S.; Yamamoto, M.; Oostinga, J. B.; Morpurgo, A. F.; Tarucha, S. Nat. Nanotechnol. 2009, 4, 383.

[97] Lui, C. H.; Li, Z. Q.; Mak, K. F.; Cappelluti, E.; Heinz, T. F. Nat. Phys. 2011, 7, 944 .

[98] Bao, W.; Jing, L.; Velasco Jr, J.; Lee, Y.; Liu, G.; Tran, D.; Standley, B.; Aykol, M.; Cronin, S. B.; Smirnov, D.; Koshino, M.; McCann, E.; Bockrath, M.; Lau, C. N. Nat. Phys. 2011, 7, 948.

[99] Liu, N.; Fu, L.; Dai, B. Y.; Yan, K.; Liu, X.; Zhao, R. Q.; Zhang, Y. F.; Liu, Z. F. Nano Lett. 2011, 11, 297.

[100] Yang, M. M.; Zhao, R. Q.; Wang, J. Y.; Zhang, L. M.; Xie, Q.; Liu, Z. F.; Liu, Z. R. J. Appl. Phys. 2013, 113, 084313.

[101] Zhang, L. M.; Yu, J. W.; Yang, M. M.; Xie, Q.; Peng, H. L.; Liu, Z. F. Nat. Commun. 2013, 4, 1443.

[102] Zhang, Y. H.; Zhou, K. G.; Xie, K. F.; Zeng, J.; Zhang, H. L.; Peng, Y. Nanotechnology 2010, 21, 065201 .

[103] Basu, D.; Gilbert, M. J.; Register, L. F.; Banerjee, S. K.; MacDonald, A. H. Appl. Phys. Lett. 2008, 92, 042114.

[104] Dutta, S.; Pati, S. K. J. Mater. Chem. 2010, $20,8207$.

[105] Son, Y. W.; Cohen, M. L.; Louie, S. G. Phys. Rev. Lett. 2006, 97, 216803. 